\title{
Pharmacokinetics of Colistin Methanesulfonate and Colistin in a Critically Ill Patient Receiving Continuous Venovenous Hemodiafiltration
}

Intravenous colistin methanesulfonate (CMS) is increasingly the last line of defense for multidrug-resistant gram-negative bacteria and is now being used as "salvage" therapy in critically ill patients $(1,7,9,11)$. CMS is converted in vivo to colistin, and these two entities have substantially different pharmacokinetics, antibacterial activities, and toxicities (7), and therefore it is important to understand the dispositions of both. Patients who are currently being treated with CMS include many in the intensive care setting with multiple organ dysfunctions and receiving renal replacement therapy (10). Goodwin et al. reported the effects of renal impairment, peritoneal dialysis, and hemodialysis on the concentrations of CMS in serum (3); unfortunately, the microbiological assays employed failed to measure colistin and CMS separately. Moreover, the efficiency of the hemodialyzer used in the 1968 report of Goodwin et al. may be different from that of current hemofilter units. To date, there is a dearth of information in critically ill patients on the clinical pharmacokinetics of CMS and the generated colistin to guide the selection of dosage regimens of CMS.

We describe here the case of a 53-year-old woman $(110 \mathrm{~kg}$; ideal body weight, $61 \mathrm{~kg}$ ) who presented with bowel perforations leading to multiple organ failure requiring continuous venovenous hemodiafiltration (CVVHDF). CVVHDF (2) was performed with 1 liter/h of dialysate and 2 liters/h of postdilution replacement fluid, producing 3 liters/h of dialysis effluent; blood flow was set at $200 \mathrm{ml} / \mathrm{min}$ through an extracorporeal circuit containing a Hospal AN69HF hemofilter. Systemic tobramycin had been ceased on day 20 of hospital admission due to decreased renal function and nebulized tobramycin $(80 \mathrm{mg}$ every $8 \mathrm{~h}$ ) was commenced. Due to an infection caused by a new multidrug-resistant Pseudomonas aeruginosa strain isolated in endotracheal aspirate, which was susceptible only to colistin and tobramycin, intravenous CMS at a dosage of 150 $\mathrm{mg}$ (equivalent to $2.46 \mathrm{mg}$ per $\mathrm{kg}$ of ideal body weight) every 24 $\mathrm{h}$ was commenced on day 24 of admission and changed to 150 $\mathrm{mg} / 48 \mathrm{~h}$ on day $38(12,14)$. Each dose was administered as a 30-min infusion. Meropenem and nebulized tobramycin were coadministered. After CMS administration on day 44, blood and effluent dialysate were collected up to $48 \mathrm{~h}$ postdose for high-performance liquid chromatography quantification of CMS (5) and colistin (4) in plasma and dialysate (accuracy within $14.1 \%$ and coefficients of variation less than $6.66 \%$ ), to permit pharmacokinetic analysis. MICs of CMS and colistin were determined by broth microdilution (13).

The concentrations of CMS and colistin in plasma and dialysate are shown in Fig. 1. The maximum concentration of colistin in plasma occurred $30 \mathrm{~min}$ after completion of the CMS infusion, consistent with relatively rapid conversion to colistin (6). The terminal half-lives of CMS and colistin in plasma were $6.83 \mathrm{~h}$ and $7.52 \mathrm{~h}$, respectively. The total clearance of CMS was $48.7 \mathrm{ml} / \mathrm{min}$ and its volume of distribution was 10.9 liters. From 0 to $8 \mathrm{~h}$ after dosing, $20.3 \%$ of the dose was recovered in the dialysate as CMS and $6.88 \%$ as colistin. CVVHDF clearances of CMS (11.2 $\mathrm{ml} / \mathrm{min})$ and colistin (11.9 $\mathrm{ml} / \mathrm{min}$ ) were similar.

The MICs of CMS and colistin against the $P$. aeruginosa isolate from the patient were $4.0 \mathrm{mg} /$ liter and $1.0 \mathrm{mg} / \mathrm{liter}$, respectively. The maximum concentrations of CMS and colistin in plasma were $23.3 \mathrm{mg} /$ liter and $1.84 \mathrm{mg} /$ liter, respectively (Fig. 1). Even without the consideration of plasma protein binding, the maximum concentration for CMS in plasma was less than $16 \times$ MIC and that for colistin was less than $2 \times$ MIC; rapid killing and significant postantibiotic effect in vitro against $P$. aeruginosa has been shown to occur above these MIC thresholds (8). In addition and importantly, the plasma con-

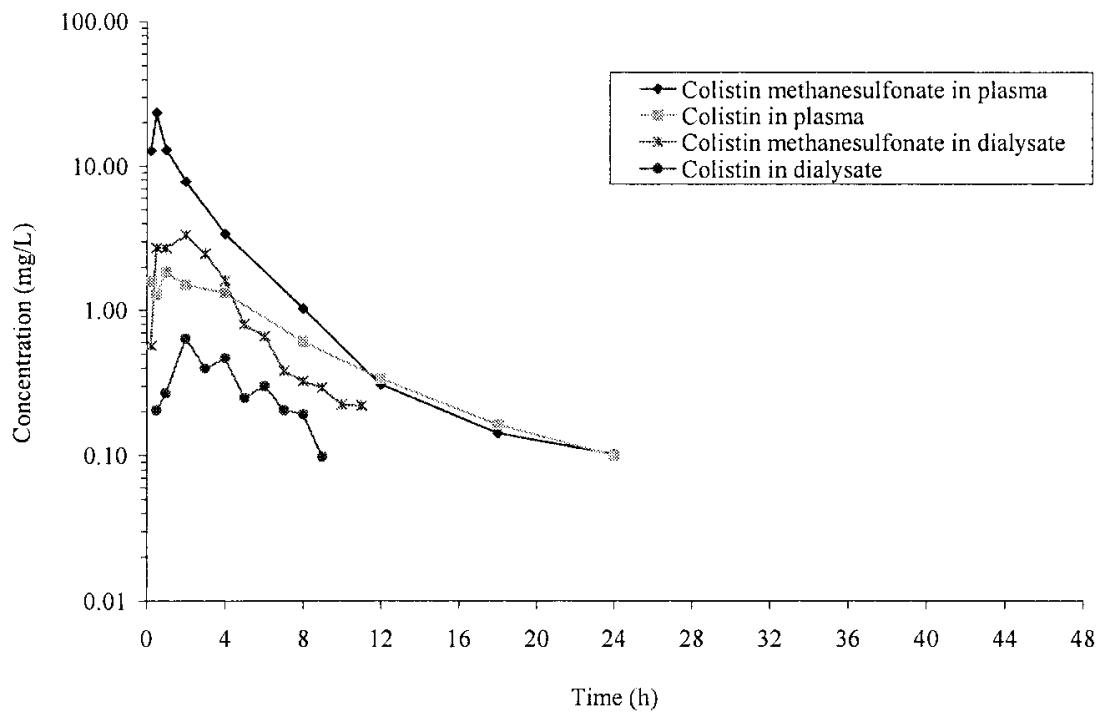

FIG. 1. Concentrations of CMS and colistin in plasma and dialysate after CMS administration (150 mg/48 h) on day 44 . Concentrations in plasma and dialysate were not quantifiable beyond $24 \mathrm{~h}$ and 9 to $11 \mathrm{~h}$, respectively. 
centrations of CMS and colistin fell below their respective MICs approximately $4 \mathrm{~h}$ following administration of CMS. Thus, plasma concentrations were below the respective MICs for a very large proportion of the 48-h dosage interval.

The product information $(12,14)$ for CMS injection is unhelpful in relation to the selection of appropriate dosage regimens in critically ill patients receiving renal replacement therapy. For patients with renal impairment, the product information suggests to essentially maintain the size of each dose while extending the dosage interval $(12,14)$, as occurred in the patient in the present study. The product information available in Australia is silent on whether CMS is cleared by dialysis (14), while the product information available in the United States claims that it is not known if CMS is cleared by dialysis (12). In the present case, we have demonstrated that both CMS and colistin are indeed cleared by CVVHDF. Unfortunately, on day 50 of admission, the patient arrested from hypovolemic and septic shock and died. Clearly, the CMS dosage regimen $(150 \mathrm{mg}$ [ $2.46 \mathrm{mg}$ per $\mathrm{kg}$ of ideal body weight] every $48 \mathrm{~h}$ ) was not appropriate for this patient. Based on the data obtained from the present case, it is likely that dosage adjustment for CMS in CVVHDF patients should be more modest (e.g., 2 to $3 \mathrm{mg} / \mathrm{kg}$ every $12 \mathrm{~h}$ ) than that employed for this patient; more definitive dosage recommendations require studies of other patients.

(This study will be presented at the 45th Interscience Conference on Antimicrobial Agents and Chemotherapy, Washington, D.C., 16 to 19 December 2005.)

We acknowledge the Australian National Health and Medical Research Council.

\section{REFERENCES}

1. Berlana, D., J. M. Llop, E. Fort, M. B. Badia, and R. Jodar. 2005. Use of colistin in the treatment of multiple-drug-resistant gram-negative infections. Am. J. Health Syst. Pharm. 62:39-47.

2. DelDot, M. E., J. Lipman, and S. E. Tett. 2004. Vancomycin pharmacokinetics in critically ill patients receiving continuous venovenous haemodiafiltration. Br. J. Clin. Pharmacol. 58:259-268.

3. Goodwin, N. J., and E. A. Friedman. 1968. The effects of renal impairment, peritoneal dialysis, and hemodialysis on serum sodium colistimethate levels. Ann. Intern. Med. 68:984-994.

4. Li, J., R. W. Milne, R. L. Nation, J. D. Turnidge, K. Coulthard, and D. W. Johnson. 2001. A simple method for the assay of colistin in human plasma, using pre-column derivatization with 9-fluorenylmethyl chloroformate in solid-phase extraction cartridges and reversed-phase high-performance liquid chromatography. J. Chromatogr. B 761:167-175.

5. Li, J., R. W. Milne, R. L. Nation, J. D. Turnidge, K. Coulthard, and J. Valentine. 2002. Simple method for assaying colistin methanesulfonate in plasma and urine using high-performance liquid chromatography. Antimicrob. Agents Chemother. 46:3304-3307.

6. Li, J., R. W. Milne, R. L. Nation, J. D. Turnidge, T. C. Smeaton, and K. Coulthard. 2004. Pharmacokinetics of colistin methanesulphonate and colistin in rats following an intravenous dose of colistin methanesulphonate. $\mathrm{J}$ Antimicrob. Chemother. 53:837-840.

7. Li, J., R. L. Nation, R. W. Milne, J. D. Turnidge, and K. Coulthard. 2005 Evaluation of colistin as an agent against multi-resistant Gram-negative bacteria. Int. J. Antimicrob. Agents 25:11-25.

8. Li, J., J. Turnidge, R. Milne, R. L. Nation, and K. Coulthard. 2001. In vitro pharmacodynamic properties of colistin and colistin methanesulfonate against Pseudomonas aeruginosa isolates from patients with cystic fibrosis. Antimicrob. Agents Chemother. 45:781-785.

9. Linden, P., S. Kusne, K. Coley, P. Fontes, D. Kramer, and D. Paterson. 2003. Use of parenteral colistin for the treatment of serious infection due to antimicrobial-resistant Pseudomonas aeruginosa. Clin. Infect. Dis. 37:E154 E60.

10. Markou, N., H. Apostolakos, C. Koumoudiou, M. Athanasiou, A. Koutsoukou, I. Alamanos, and L. Gregorakos. 2003. Intravenous colistin in the treatment of sepsis from multiresistant Gram-negative bacilli in critically ill patients. Crit. Care 7:R78-R83.

11. Michalopoulos, A., S. Kasiakou, E. Rosmarakis, and M. Falagas. 2005. Cure of multidrug-resistant Acinetobacter baumannii bacteraemia with continuous intravenous infusion of colistin. Scand. J. Infect. Dis. 37:142-145.

12. Monarch Pharmaceuticals. 2000. Coly-Mycin M Parenteral. Package insert. Monarch Pharmaceuticals, Bristol, Tenn.

13. National Committee for Clinical Laboratory Standards. 1992. Methods for determining bactericidal activity of antimicrobial agents; tentative guideline M26-T. National Committee for Clinical Laboratory Standards, Villanova, $\mathrm{Pa}$.

14. Pfizer. 2003. Coly-Mycin M Parenteral. Package insert. Pfizer Australia Pty. Ltd., West Ryde, NSW, Australia.

\section{J. Li \\ C. R. Rayner}

R. L. Nation*

Facility for Anti-infective Drug Development and Innovation

Victorian College of Pharmacy

Monash University

Melbourne, Australia

R. Deans

R. Boots

N. Widdecombe

A. Douglas

J. Lipman

Burns, Trauma and Critical Care Research Centre

Department of Anaesthesiology and Critical Care

University of Queensland

Brisbane, Australia

*Phone: 61399039061

Fax: 61399039629

E-mail: roger.nation@vcp.monash.edu.au 